\title{
INCUBACIÓN SEMIARTIFICIAL DE HUEVOS DE TARICAYA Podocnemis unifilis TROSCHEL (1848), EN DOS TIPOS DE SUSTRATOS
}

\author{
Melba del Rocío CORREATANG ${ }^{1}$, Lucy ARMAS MAITAHUARI $^{2}$ \\ 1 Instituto de Investigaciones de la Amazonía Peruana (IIAP), Programa de Investigación en Biodiversidad Amazónica \\ (PIBA). Iquitos, Perú, rcorrea@iiap.org.pe \\ 2 Reserva Nacional Pacaya Samiria - Programa de Educación Ambiental.
}

\section{RESUMEN}

Este estudio tuvo como objetivo evaluar el porcentaje de eclosión de los huevos de la taricaya Podocnemis unifilis Troschel (1848) en los bancos de incubación semiartificial. La incubación de los huevos fue realizada de setiembre a diciembre del 2014 en playas artificiales, utilizando dos tipos de sustratos (arena de cantera y arena de playa). Se evaluó el periodo de incubación (periodo comprendido entre la fecha de postura hasta la salida de los neonatos), la temperatura promedio y la tasa de eclosión en cada tipo de sustrato, con tres repeticiones cada una. El periodo de incubación varió de 74 a 88 días para arena de playa y de 77 a 90 días para arena de cantera. La temperatura promedio para arena de cantera fue de $26,9^{\circ} \mathrm{C}$ y para arena de playa de 27,1 ${ }^{\circ} \mathrm{C}$. La tasa de eclosión promedio fue diferente para ambos sustratos (arena de playa $=14.7, \pm 3.2$; arena de cantera $=8, \pm 2.6)$, Sin embargo, estas diferencias no fueron estadísticamente significativas ( $p>0.05)$.

PALABRAS CLAVE: Incubación semiartificial, temperatura promedio, periodo de incubación, tasa de eclosión, Podocnemis unifilis, taricaya.

\section{SEMIARTIFICIAL INCUBATION OF EGGS OF TARICAYA Podocnemis unifilis TROSCHEL (1848) IN TWO TYPES OF SUBSTRATES}

\section{ABSTRACT}

The aim of this study was to evaluate the hatching percentage of hatchings of the taricaya Podocnemis unifilis Troschel (1848) in semi-artificial incubation banks. Eggs were incubated from September to December 2014 in artificial beaches using two types of substrates (river beach sand and quarry sand). The incubation period (the period between the date of egg laying until egg hatching), the mean temperature and the hatching rate for each type of substrate, with three repetitions were evaluated. The incubation period on sandy beach varied from 74 to 88 days and from 77 to 90 days on sand quarry. The average temperature for sand quarry was $26.9^{\circ} \mathrm{C}$ and $27.1^{\circ} \mathrm{C}$ for sand beach. The average hatching rate was different for both substrates (sand beach $=14.7 \pm 3.2$; sand quarry $=8 \pm 2.6$ ), but these values were not significantly different ( $\mathrm{t}$ student Test; $\mathrm{p}>0.05)$.

KEYWORDS: Semiartificial incubation, mean temperature, incubation period, hatching rate, Podocnemis unifilis, taricaya. 


\section{INTRODUCCIÓN}

Es muy probable que los quelonios no hayan sido nunca un grupo tan numeroso como el de otros tetrápodos, pero considerando su relativamente baja diversidad actual preocupa que alrededor del $50 \%$ de sus especies se encuentren bajo algún grado de amenaza. Esto confiere a las tortugas el dudoso privilegio de ser los vertebrados más presionados, superando inclusive a los anfibios (Van Dijk et al., 2012). Es así que en el ámbito mundial, las tortugas se encuentran en serios problemas; son cazadas, sacrificadas, consumidas y vendidas en cantidades desmedidas. Los huevos, crías y adultos son usados como comida, mascotas y en medicina. Son explotadas de forma indiscriminada y $\sin$ consideraciones de sustentabilidad, por lo que muchas especies se encuentran al borde de la extinción y otras ya se han perdido (Turtle Conservation Fund, 2002). La taricaya Podocnemis unifilis no escapa a esta situación, en los últimos años el aumento de la presión sobre sus poblaciones naturales ha llevado a que esta especie sea considerada vulnerable por la IUCN 2009 (PinedaCatalán et al., 2012).

La taricaya Podocnemis unifilis se caracteriza por presentar un caparazón cúpula con solamente una quilla mediana que se evidencia más en los especímenes inmaduros, los machos son menores que las hembras (Vogth, 2008). Según Soini (1999), esta especie presenta el mayor registro en recolección, eclosión y liberación que cualquier otra entre los quelonios acuáticos de la Amazonía peruana. Pero no siempre fue así, en décadas pasadas, debido al declive de la charapa comenzó a darse mayor presión sobre otras dos especies de interés: taricaya Podocnemis unifilis y cupiso Podocnemis sextuberculata, situación que se ha desencadenado igualmente en países como Perú y Brasil (Fachín - Terán, 1999), es así que la presión ejercida por el hombre ha conllevado a su disminución y desaparición en muchas zonas, especialmente en aquellos lugares cercanos a las comunidades que rodean a la Reserva Nacional Pacaya-Samiria, en Perú.

La taricaya tal vez sea la especie más investigada, dentro del género Podocnemis, en lo que se refiere a su ecología reproductiva. Se realizaron una serie de trabajos en la Amazonía brasilera (Vanzolini, 1977; Souza \& Vogt, 1994; Vogt, 2012), de Colombia (Medem, 1964; Foote, 1978; Paéz, 1995; Paéz \& Bock, 1997, 1998), de Venezuela (Thorbjarnarson et al., 1993), de Bolivia
(Caballero, 1996) y del Perú (Ponce, 1979; FachínTerán, 1992, 1993; Fachín-Terán et al., 1997; Soini, 1995a, 1995b, 1995c; Soini \& Coppula, 1995; Soini \& Soini, 1995a, 1995b, 1995c; Soini, 1996, 1997). En los últimos años se registraron informaciones sobre el estado de conservación molecular, así como parasitológica, de sus poblaciones naturales en la Amazonía peruana (Pineda-Catalán et al., 2012, Pineda-Catalán et al., 2013). Los únicos registros sobre reproducción de esta especie fueron reportados para la Amazonía brasilera y abordan aspectos de nidificación (Thorbjarnarson \& Silveira, 1996, citado en Fachín - Terán \& Matheus, 2003; Vogt, 2008). Sin embargo, algunos aspectos sobre la nidificación en bancos semiartificiales con diferentes sustratos todavía son poco conocidos en esta parte de la región amazónica.

En el Perú, debido a la categorización de $P$. unifilis como especie vulnerable, diversas instituciones vienen aunando esfuerzos para incrementar sus poblaciones naturales a través de programas de repoblamiento, para lo cual vienen realizando trabajos de incubación de huevos en playas artificiales con la participación de algunas instituciones educativas de zonas rurales, periurbanas y urbanas (SERNANP, 2010). En la Reserva Nacional Pacaya Samiria también se involucra a los pobladores de las comunidades (conformados actualmente en grupos de manejo) en la aplicación de técnicas de propagación y protección de los lugares de desove, dando muy buenos resultados, lo que confirma que mediante acciones acertadas de manejo, el recurso puede ser aprovechado de forma sostenible, pues constituyen una fuente tradicional de carne y huevos para la población (AECI, 2005). En condiciones artificiales la técnica de exposición al sol para la producción de neonatos de $P$. unifilis, utilizando dos tipos de sustratos (arena de cantera y arena de playa), se presenta como una alternativa para diversificar las técnicas de incubación de esta especie en los lugares donde ya no existen, dentro de programas de manejo sostenido.

El presente estudio pretende contribuir al manejo y conservación de esta especie a través de la generación de conocimientos sobre la eclosión de la taricaya en condiciones semi artificiales, utilizando dos diferentes sustratos, lo que permitirá un mayor desarrollo de los programas de educación ambiental en las diferentes instituciones educativas de la región amazónica, contribuyendo a los propósitos de la Educación y las Políticas de Estado del Perú al 2021.(PEN 2010). 


\section{MATERIAL Y MÉTODOS}

\section{Área del estudio}

El trabajo fue ejecutado en las instalaciones de la sede central del Instituto de Investigaciones de la Amazonía Peruana (IIAP), ubicado en la ciudad de Iquitos, departamento de Loreto, Amazonía peruana. La temperatura media anual del ambiente es de $26^{\circ} \mathrm{C}$, con oscilaciones anuales que no sobrepasan $\operatorname{los} 5^{\circ} \mathrm{C}$. La precipitación promedio anual es superior a 2,600 mm (Kalliola \& Flores 1998). Durante el año se presentan dos estaciones, la estación seca que va de julio a setiembre, con una precipitación alrededor de $229 \mathrm{~mm}$ y una temperatura promedio de $32{ }^{\circ} \mathrm{C}$ y la estación lluviosa que va de noviembre a mayo con una precipitación de $300 \mathrm{~mm}$ y una temperatura promedio de $26^{\circ} \mathrm{C}$ (Climate Classification and Climatic Regions of the World, 2012)

\section{Construcción de los bancos de incubación}

Para la incubación de los huevos, se construyeron tres cajones o bancos de incubación artificial tipo "parrilla" (BIA), separados por un $1 \mathrm{~m}$ de distancia cada uno. Los BIA fueron construidos de madera, de $1 \mathrm{~m}$ de largo, $1 \mathrm{~m}$ de ancho y $0.70 \mathrm{~m}$ de alto; el área de cada BIA fue subdividida en dos partes de $1 \mathrm{~m}$ x $50 \mathrm{~cm}$ cada una, obteniéndose seis espacios de incubación, tres para el sustrato de arena de cantera $(\mathrm{Ac})$ y tres para el sustrato de arena de playa (Ap).

La arena de cantera (Ac) provino de la carretera Iquitos - Nauta, fue de textura gruesa, de color gris oscuro a blanco, suelta, químicamente de reacción fuertemente ácida ( $\mathrm{pH} 4.59)$, baja en contenido de materia orgánica, fósforo y potasio (IIAP, 2012). La arena de playa (Ap) provino del río Amazonas, de textura muy fina y pequeña, de color gris oscuro, alta en contenido de materia orgánica, nitrógeno, fósforo y potasio (Rodríguez, 1990).

En cada espacio de incubación se colocó $37 \mathrm{~m}^{3}$ de arena limpia de impurezas, como raíces, hojarasca y hierbas. Para proteger los huevos de predadores naturales como hormigas, cada BIA fue colocado a $40 \mathrm{~cm}$ de altura, sobre soportes de madera protegido con aceite quemado.

\section{Reanidación}

Para el experimento, se colectaron huevos de seis nidos de "taricaya" de la misma fecha de postura (30 de agosto del 2014), provenientes de playas naturales, localizadas dentro de la Reserva Nacional Pacaya - Samiria. En los BIA, los huevos fueron colocados en hoyos (nidos seminaturales) de $15 \mathrm{~cm}$ de profundidad, $10 \mathrm{~cm}$ de diámetro y cubiertos con una capa de arena de $5 \mathrm{~cm}$, tratando de simular un nido natural (Soini 1999). En cada BIA se realizaron seis hoyos y en cada hoyo se colocaron 20 huevos, con tres repeticiones por cada tipo de sustrato.

Antes de reanidar, se realizó la selección de los huevos viables (presentan una mancha blanquecina) descartando huevos rotos, deformados y no viables (Soini, 1999). En los nidos seminaturales se colocaron placas, con el número de huevos, la fecha de postura y reanidación.

Durante el periodo de incubación fue registrada tres veces al día (7:00, 13:00 y 19:00 horas) la temperatura de cada nido a $9 \mathrm{~cm}$ de profundidad. Estas mediciones fueron realizadas con ayuda de un termómetro digital marca HANNA, modelo Checktemp 1 , con un rango -50 a $150{ }^{\circ} \mathrm{C}$.

\section{Evaluación post-reanidación}

Al finalizar el experimento los huevos se clasificaron de acuerdo a los criterios de la UICN (2013): huevos eclosionados (huevos en donde los neonatos lograron salir de él y emerger a la superficie), huevos eclosionados no emergentes (huevos en donde los neonatos lograron salir de él, pero que no lograron emerger a la superficie), huevos no eclosionados (huevos que no eclosionaron pero que contienen embriones), huevos infértiles (huevos que no contienen embrión).

Para el registro de la información se tuvo en consideración el periodo transcurrido entre la postura y la salida del neonato del huevo (Hildebrand et al., 1997), que comprende la fecha de postura, días de reanidación y eclosión para todos los nidos por sustrato.

\section{Procesamiento de la información}

Los datos fueron analizados mediante estadística descriptiva (media, desviación estándar, T-test Correlación de Pearson). Para calcular la tasa de eclosión (Te) se utilizó la siguiente fórmula: T.e. $=(\mathrm{a} / \mathrm{b})$ x100 donde: (a) es número de huevos eclosionados por sustrato, (b) es el número de huevos reanidados por sustrato. Para comparar la eclosión de huevos entre los dos tipos de sustrato se empleó el T-test, para relacionar con la temperatura la Correlación de Pearson.

\section{RESULTADOS Y DISCUSIÓN}

Durante el estudio se ha obtenido un promedio de nacimientos de $14.7( \pm 3.2)$ en arena de playa $(73 \%$ del total reanidados) y $8( \pm 2.6)$ en arena de cantera, lo que representa un $40 \%$ del total de reanidados en este sustrato. Si bien, estas diferencias en la tasa de eclosión entre ambos sustratos no fueron estadísticamente significativas ( $p>0.05$ ), el $73 \%$ de 
nacimientos en arena de playa es importante en términos prácticos para la obtención de mayores eclosiones (Tabla 2 y Figura 1). Este valor se aproxima al 78\% reportado por Ushiñahua (1995), para eclosión de taricaya utilizando sustrato de arena negra pura; asimismo, este porcentaje de eclosión se encuentra dentro del rango (70 a $80 \%$ ) reportado por Soini (1995) para nidos transplantados de taricaya.

En tanto que la tasa de eclosión del $40 \%$ en el sustrato de arena de cantera fue superior a lo reportado por Fachín \& Acosta (1994); quienes obtuvieron una tasa de eclosión del $27.6 \%$ utilizando como sustrato arena blanca en bancos de incubación artificial. Sin embargo, la tasa de eclosión obtenida en el presente estudio para ambos tipos de sustratos (promedio 56.5\%), es baja en comparación a lo obtenido en otros años (entre 58 a $62 \%$ ). Esta disminución en la tasa de eclosión puede estar relacionada a factores climáticos como cambios repentinos de temperatura y constantes precipitaciones; que se evidenciaron en el periodo de experimentación (octubre a noviembre del 2014), así como a la mala manipulación de los huevos durante todo el proceso (RNPS, 2005).
La figura 1 muestra que el promedio de huevos eclosionados no emergentes del sustrato arena de cantera fue mayor (5) que el de arena de playa (2.3), y que el promedio de huevos infértiles fue relativamente semejante entre ambos sustratos $($ arena de playa $=2$ y arena de cantera $=2.7)$. En esta figura podemos observar además que los promedios de huevos no eclosionados fueron muy diferentes entre arena de cantera y arena de playa (4.3 y 1 respectivamente).

El periodo de incubación para el sustrato arena de playa fue de 74 a 88 días y para el sustrato de arena de cantera entre 77 a 90 días. Estos valores encontrados fueron mayores a los obtenidos por Fachín \& Matheus (2003) quienes reportaron un tiempo medio de incubación de huevos de 61.7 ( \pm 3 días) para el sustrato de arena y (72.8 \pm 0.44 días) con respecto a cinco nidos incubados en sustrato arcilloso; y Rengifo (1997) que obtuvo un periodo de incubación de 61 a 74 días utilizando arena de playa pura negra. En lo que respecta al sustrato de arena de cantera nuestro periodo de incubación varió de 77 a 90 días, valores que difieren a lo reportado por Fachín \& Acosta (1994) de 111.8 días utilizando arena blanca.

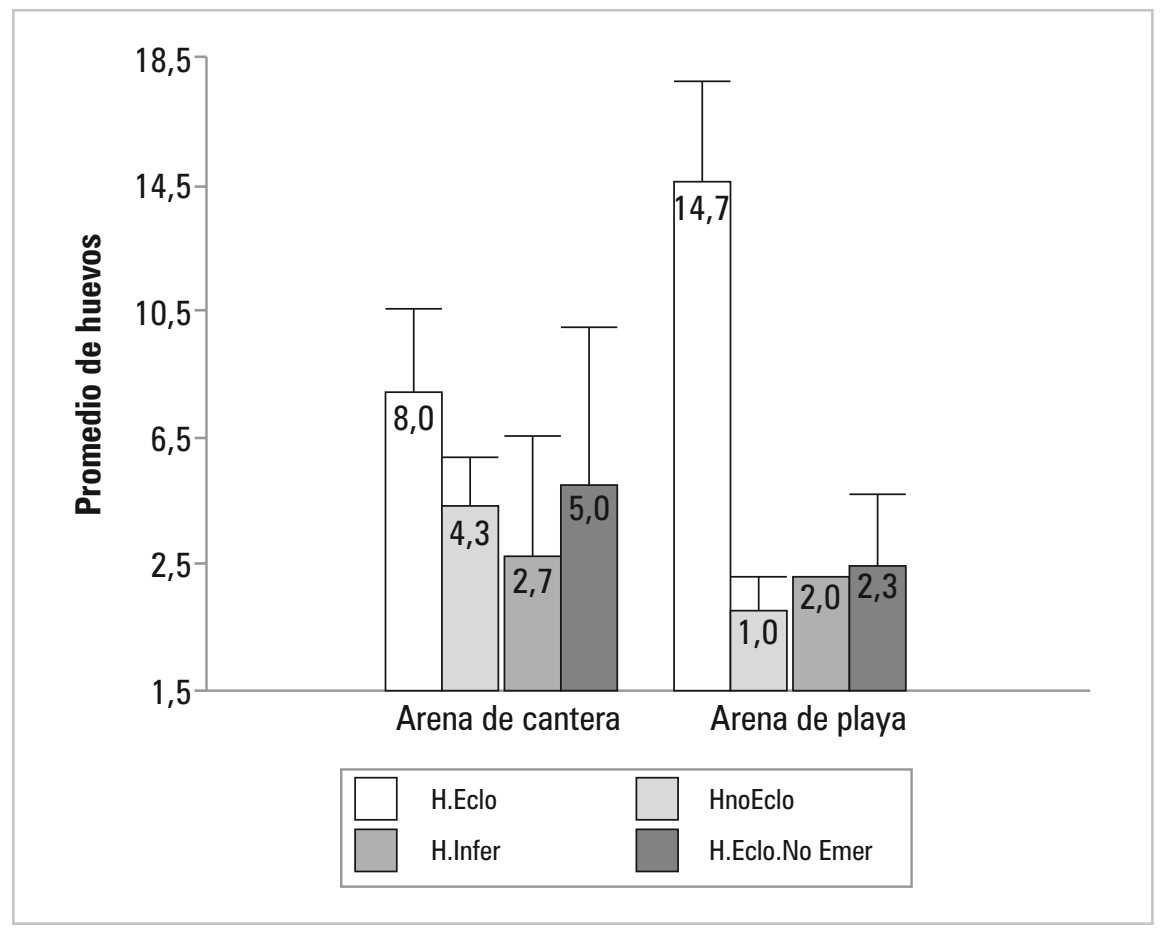

Figura 1. Tasa promedio de eclosión de huevos de Taricaya, reanidados en arena de cantera y arena de playa. 
Tabla 1. Temperatura registrada en el sustrato arena de cantera y arena de playa utilizada en los nidos artificiales de taricaya Podocnemis unifilis.

\begin{tabular}{lcc}
\hline \multicolumn{1}{c}{ Periodos } & Arena de cantera & Arena de playa \\
\hline Mañana (07:00 horas) & 25.4 & 25.5 \\
\hline Tarde (13:00 horas) & 29.4 & 29.8 \\
Noche (19:00 horas) & 25.8 & 26.0 \\
Promedio & 26.9 & 27.1 \\
\hline
\end{tabular}

Asimismo el periodo de incubación obtenido por los dos sustratos es mayor en comparación a lo reportado por RNPS (2005) y Vogt (2008), quienes mencionaron que el periodo de incubación en circunstancias normales es de 72 a 97 días. Sin embargo nuestros valores se encuentran dentro del rango reportado por Soini \& Coppula (1995), quienes refieren que el rango de incubación es de 55 a 97 días en playas de arena con mucha exposición al sol.

La fecha de salida de los neonatos, contando desde la fecha de postura (30 de agosto) para el sustrato arena de playa inició entre el 13 y el 25 de noviembre, teniendo el mayor número de salidas el día 13 con una temperatura promedio de $28.2^{\circ} \mathrm{C}$. En el sustrato arena de cantera la salida de los neonatos fue del 16 hasta el 29 de noviembre, teniendo el mayor número de salidas los días 16 y 17 con temperaturas promedio de $27.1^{\circ} \mathrm{C}$ y $25.6{ }^{\circ} \mathrm{C}$ respectivamente. Las temperaturas promedios registradas fueron relativamente semejantes entre los sustratos, $27,1^{\circ} \mathrm{C}$ para el sustrato arena de playa y de $26,9^{\circ} \mathrm{C}$ para el sustrato arena de cantera (Tabla 1). Estas pequeñas diferencias en la temperaturas de estos dos sustratos pueden estar relacionados con el color de los mismos, es decir, la arena de las playas de los ríos, debido a que por su color oscuro (conferido por el limo y algunos fragmentos de rocas de color negro como la pizarra, el basalto y el color verde oscuro como la roca limonita) conservan relativamente mejor el calor, que la arena de las canteras. Teniendo en cuenta que las altas temperaturas disminuyen el periodo de incubación (Páez \& Bock, 1998 citado por García, 2005), las diferencias entre las temperaturas promedios del sustrato arena de playa y arena de cantera no fueron lo suficientemente grandes para diferenciar los periodos de incubación entre los sustratos.
Estos resultados nos permiten concluir que la utilización de arena de playa y arena de cantera como sustrato en nidos artificiales no influyen significativamente en la eclosión de la taricaya Podocnemis unifilis, además que la temperatura promedio de ambos sustratos fue relativamente semejante por lo que no se observó diferencias en los periodos de eclosión.

\section{AGRADECIMIENTOS}

Los autores agradecen a Elvis Paredes y Harrison García por el apoyo prestado durante el desarrollo de este estudio. A Carmen García por la revisión y aporte al manuscrito.

\section{BIBLIOGRAFÍA CITADA}

AECI. 2005. Plan de Manejo para el Aprovechamiento de "Taricaya" (Podocnemis unifilis) en la cuenca del Yanayacu Pucate. Iquitos, Perú. 48pp.

Climate Classification and Climatic Regions of the World PhysicalGeography.net. (http://www. physicalgeography.net/fundamentals/7v.html)

Fachín-Terán, A; Matheus, E. 2003. Reproducción de la taricaya Podocnemis unifilis Troschel 1848 (TESTUDINES: PODOCNEMIDIDAE) en la várzea del medio Solimões, Amazonas, Brasil. Ecología Aplicada, 2(1): 125-132.

Fachín-Terán, A. 1999. Ecología de P. sextuberculata (Testudines, Pelomedusidae) $\mathrm{Na}$ reserva de desenvolvimiento sustentável Mamirauá, Amazonas. Brasil. Tesis de Postgrado de la Universidad Federal do Amazonas UA, Instituto de pesquisas da amazonía-INPA. Manaos. 189pp. 

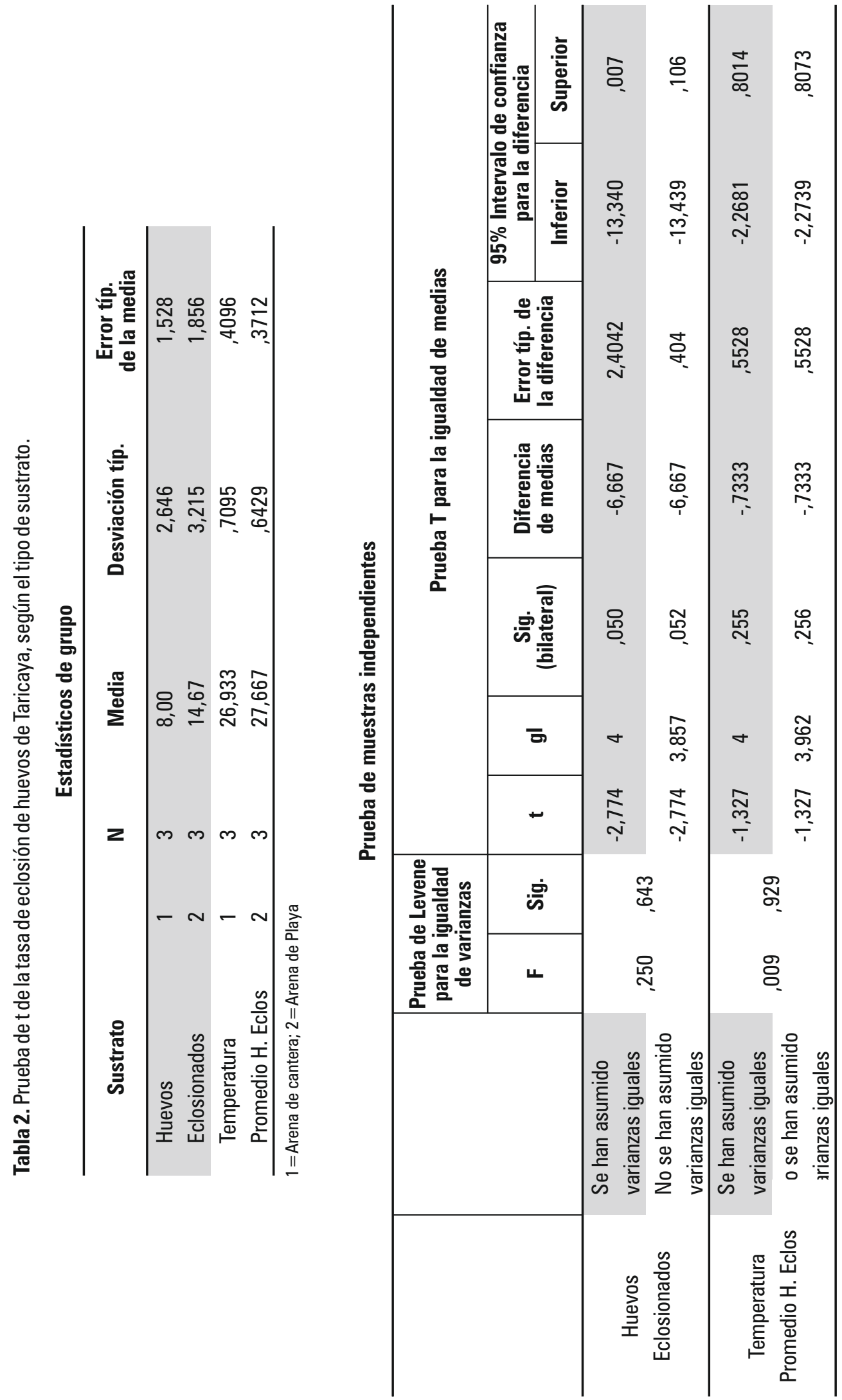
Fachín-Terán, A.; Acosta, A.; Vilchez I.; Taleixo, G.1997. Reproducción de la taricaya Podocnemis unifilis (Reptilia: Testudines) en cautiverio,Iquitos, Perú. In: Tula G. Fang, Richard E.Bodmer, Rolando Aquino y Michael H. Valqui (eds.). Manejo de Fauna Silvestre en la Amazonía.p.185-189.

García, N. 2005. Biología reproductiva y conservación de las tortugas "charapa" Podocnemis expansa, "cupiso" Podocnemis sextuberculata y "taricaya" Podocnemis unifilis. Tesis para optar Título de Ecóloga. En las playas aledañas al Municipio de Puerto Nariño (Amazonas). Pontifica universidad javeriana facultad de estudios ambientales y rurales carrera de pregrado en Ecología. Perú. 209pp.

Hildebrand, Von P.; Bermudez, N.; Peñuela, M.C. 1997. La Tortuga charapa (Podocnemis expansa) en el río Caquetá Amazonas, Colombia. Aspectos de la biología reproductiva y técnicas para su manejo. Disloque. 152pp.

IIAP, 2012. Suelos y Capacidad de Uso Mayor de las Tierras. Microzonificación Ecológica y Económica del área de influencia de la carretera Iquitos-Nauta. $115 \mathrm{pp}$.

Kalliola, R.; Flores, S. 1998. Geoecología y Desarrollo Amazónico. Estudio integrado en la zona de Iquitos, Perú. Turun Yliopiston Julkaisuja, Turku.544 pp.

MINISTERIO DE EDUCACIÓN.2010. Proyecto Educativo Nacional. Resolución Ministerial $\mathrm{N}^{\circ}$ 215_2001_PE.

Pineda-Catalan, O.; Mendez, M.; Gleizer, A.; García-Dávila, C.; Aguirre, A.; Pinedo-Vasquez, M.; Amato, G. 2012. Conservation genetics of harvested river turtles, Podocnemis expansa and P. unifilis, in the Peruvian Amazon: All roads lead to Iquitos. Mitochondrial DNA, 23(3) 230238.

Pineda-Catalan, O.; Perkins, S. L.; Peirce, M.A.; Engstrand , R.; Garcia-Davila, C.; PinedoVasquez, M.; Alonso- Aguirre, A 2013. Revision of Hemoproteid Genera and Description and Redescription of Two Species of Chelonian Hemoproteid Parasites. Journal of Parasitology, 99(6):1089-1098.

RNPS. 2010. Plan de manejo para el aprovechamiento de "taricaya" (Podocnemis unifilis) en la cuenca del Yanayacu-Pucate, Reserva Nacional Pacaya-Samiria. Iquitos Perú. 73pp.

RNPS. 2005. Plan de manejo para el aprovechamiento de "taricaya" (Podocnemis unifilis) en la cuenca del Yanayacu-Pucate, Reserva Nacional Pacaya-Samiria. Grupos organizados de manejo. Iquitos-Perú. 51pp.
Rengifo F. 1997. Experiencia de manejo comunal de "taricaya" Podocnemis unifilis en la comunidad de Manco Capac R.N.P.S. Pro-NaturalezaPerú.9pp

Rodríguez, F. IIAP, 1990. Los suelos de áreas inundables de la Amazonia peruana: Potencial, limitaciones y estrategia para su investigación. Folia Amazónica, 2: 15-25.

SERNANP 2010. Crianza de Quelonios acuáticos de las especies Podocnemis unifilis "Taricaya" y Podocnemis expansa "Charapa", para fomentar y contribuir a la Educación Ambiental en los centros educativos de la ciudad de Yurimaguas y de la comunidad Jorge Chávez- Perú. 13pp.

Soini, P. 1994. Ecología reproductiva de la taricaya (Podocnemis unifilis) en el río Pacaya, Perú. Folia Amazónica, 6:105-124.

Soini, P.; Coppula, M. 1995. Estudio, reproducción y manejo de los quelonios del género Podocnemis (charapa, cupiso y taricaya) en la cuenca del Pacaya, río Pacaya, Loreto-Perú. Informe $\mathrm{N}^{\circ} 2$. In:Soini, P., A. Tovar y U. Valdez (ed.), Reporte Pacaya-Samiria. Investigaciones en Cahuana:1980-1994, p. 3-30. CDCUNALM/FPCN/TCN. Lima, Perú.

Soini, P. 1999. Un manejo para el manejo de Quelonios acuáticos en la Amazonía Peruana (charapa, taricaya y cupiso). Instituto de Investigaciones de la Amazonía Peruana. Iquitos. 68pp.

Turtle Conservation Fund. 2002. A Global Action Plan for Conservation of Tortoises and Freshwater Turtles. Strategy and Funding. Prospectus 2002-2007. Conservation International / Chelonian Research Foundation. Washington, DC, EEUU. 30pp.

UICN 2013. IUCN Red List of Threatened Species. Version 2013.1 (www.iucnredlist.org/) Downloaded/on 18 Diciembre 2013.

Ushiñahua, M. 1995. Experiencia de manejo de Taricaya con la comunidad de Manco Capac. RNPS.PRO-NATURALEZA.PERU.6pp

Van Dijk, P.P et al. 2012. Turtles of the world, 2012 update: Annotated checklist of taxonomy, synonymy, distribution, and conservation status. Chelonian Research Monographs 5: 000.243000.328 (doi: 10.3854/crm.5.000.checklist. v5.2012,www.iucn-tftsg.org/cbftt/)

Vogt, R. 2008. Tartarugas da Amazonia. Gráfica Biblos, Lima, Perú. 104pp.

Recibido: 16 de setiembre del 2015

Aceptado para publicación: 29 de octubre del 2015 
